\title{
Puerarin inhibits M2 polarization and metastasis of tumor-associated macrophages from NSCLC xenograft model via inactivating MEK/ERK 1/2 pathway
}

\author{
HONGGANG KANG ${ }^{1}$, JING ZHANG ${ }^{1}$, BAOZHONG WANG ${ }^{1}$, MEIRONG LIU ${ }^{1}$, \\ JUN ZHAO $^{1}$, MENGXIANG YANG ${ }^{1}$ and YINGXUE $\mathrm{LI}^{2}$ \\ Departments of ${ }^{1}$ Oncology and ${ }^{2}$ Pathology, Liaocheng People's Hospital, Liaocheng, Shandong 252004, P.R. China
}

Received September 29, 2016; Accepted December 9, 2016

DOI: $10.3892 /$ ijo.2017.3841

\begin{abstract}
Non-small cell lung carcinoma (NSCLC) metastasis is responsible for most of cancer-related mortality. The tumor associated macrophages (TAMs) are known to be crucial cells in lung cancer and are usually divided into two antagonistic types, M1 and M2. Puerarin has a wide spectrum of pharmacological properties. The present study explores puerarin on macrophage polarization and metastasis of NSCLC. The results demonstrated that puerarin inhibited tumor growth and tumor volumes in NSCLC xenograft model, increased M1 markers [CD197 ${ }^{+}$, inducible nitric oxide synthase $\left.\left.(\mathrm{iNOS})^{+}, \mathrm{CD} 40^{+}\right)\right]$and reduced $\mathrm{M} 2$ markers $\left(\mathrm{CD} 206^{+}\right.$, Arg- $1^{+}$and $\left.\mathrm{CD} 163^{+}\right)$. Besides, puerarin elevated the level of pro-inflammatory cytokine interferon (IFN)- $\gamma$, tumor necrosis factor (TNF)- $\alpha$ and interleukin (IL)-12, decreased the expression of pro-tumor cytokines IL-10, IL-4 and transforming growth factor (TGF)- $\beta$. To explore whether puerarin directly acts on macrophages, we purified macrophages from NSCLC model, the results showed that puerarin inhibited macrophages polarized to M2 phenotype and did not require the auxiliary of other cells. In addition, puerarin suppressed the invasion and migration of NSCLC macrophages, restrained the expression of angiogenesis factors. Puerarin also inhibited the activation of mitogen-activated extracellular signal-regulated
\end{abstract}

Correspondence to: Dr Jing Zhang, Department of Oncology, Liaocheng People's Hospital, 67 Dongchang West Road, Liaocheng, Shandong 252004, P.R. China

E-mail: doczhangjing@sina.com

Abbreviations: NSCLC, non-small cell lung carcinoma; TAMs, tumor associated macrophages; iNOS, inducible nitric oxide synthase; IFN- $\gamma$, interferon- $\gamma$; TNF- $\alpha$, tumor necrosis factor- $\alpha$; IL, interleukine; TGF- $\beta$, transforming growth factor- $\beta$; VEGF, vascular endothelial growth factor; MMP-9, matrix metalloproteinase-9; ICAM-1, intercellular adhesion molecule-1; MEK, mitogenactivated extracellular signal-regulated kinase; ERK, extracellular signal-regulated kinase

Key words: non-small cell lung carcinoma, metastasis, tumor associated macrophages, cytokines, extracellular signal-regulated kinase kinase (MEK)/extracellular signal-regulated kinase (ERK) $1 / 2$ pathway through inhibition of ERK nucleus translocation. Finally, IL-4 induced M2 macrophage polarization and metastasis were partially offset by puerarin through inactivating the MEK/ERK 1/2 pathway. Taken together, this study validated that puerarin is able to skew macrophage populations back to $\mathrm{M} 1$ subsets to stimulate antitumor effects and suggests puerarin is a negative metastatic regulator of NSCLC.

\section{Introduction}

Non-small cell lung carcinoma (NSCLC) is a leading cause of cancer death worldwide, and represents close to $90 \%$ of all lung cancers with a poor prognosis. The progression of lung cancer is a complex and multistep process where several mechanisms such as transformation, hypoxia, invasion, migration and metastasis are known to be the main hallmarks, especially metastasis, which is responsible for $90 \%$ of cancer-related mortality (1). Clinical data have shown that most lung cancer patients eventually suffer relapse and/or metastasis after complete excision of the cancer, even if they were at stage I-A (2). Research on NSCLC metastasis has recently expanded to tumor-microenvironment, which include the stromal cellular compartment and extra-cellular matrix components. Metastasis (from initial angiogenesis, intravasation, to extravasate into a distant tissue) is an inefficient process and few released cancer cells complete the entire process, micro-environmental interactions assist each of these steps (3). It is necessary to better understand the mechanisms by which tumor cells co-operate with the micro-environment.

Together with other immune cells, tumor cells and interstitial cytokines, the tumor associated macrophages (TAMs) can be brought to form a tumor immune microenvironment (4). TAMs refer to a kind of macrophages which are migrated and infiltrated in local tumor during the occurrence and development of tumors (5). TAMs are known to be crucial cells in lung cancer as they are in close proximity to tumor cells compared to other stromal cells (1). According to the activation type of macrophage, it can be divided into two main types M1 and M2. It is generally considered that these two types of macrophages are antagonistic $(6,7)$. M1 phenotype is activated by interferon (IFN)- $\gamma$, LPS and tumor necrosis factor (TNF)- $\alpha(8,9)$. This 
phenotype is associated with the expression of interleukin (IL)12 , TNF- $\alpha$ and inducible nitric oxide synthase (iNOS)(10,11) and also with extended survival time in NSCLC patients (12). M2 phenotype possesses effects of inflammation inhibition by producing anti-inflammatory cytokines such as IL-4 and IL-10 (7,11,13). This phenotype can promote tumor growth and sustain tumor survival (14). They also play a vital role in increasing angiogenesis via VEGF, which is a prominent mediator of angiogenesis (15). Given the above, the inducing of M1 and inhibition of M2 polarization may help alleviate the metastasis of NSCLC.

Puerarin [4H-1-benzopyran-4-one, 8 - $\beta$-D-glucopyranosyl7-hydroxy-3-(4-hydroxyphenyl), $\mathrm{C}_{21} \mathrm{H}_{20} \mathrm{O}_{9}$ ] is the major bioactive ingredient isolated from the root of traditional Chinese medicine Ge-gen (Radix Puerariae, RP) (16). Due to its wide spectrum of pharmacological properties (e.g., cardioprotection, neuroprotection, antioxidant, anticancer, alleviating pain and inhibiting alcohol intake), puerarin has been widely used in the treatment of various deseases, including cardiovascular, diabetes, Parkinson's disease, endometriosis and cancer (17). The antitumor activity of puerarin has been reported on human lung carcinoma A549 cell line (18). However, the effect of puerarin on NSCLC metastasis has rarely been reported.

The present study applied puerarin in the context of NSCLC, we found that puerarin reduced the tumor growth in NSCLC xenograft model. Besides, puerarin acted directly on macrophages to inhibit macrophage polarized to M2 phenotype and suppressed cell invasion and migration. These inhibition effects may function through inactivating MEK/ERK 1/2 pathway. Taken together, our results suggest that puerarin may provide novel insight into the mechanism and treatment of NSCLC.

\section{Materials and methods}

NSCLC xenografts. All the animals involved in the present study were purchased from the the Institute of Zoology, Chinese Academy of Medical Sciences (NOD/SCID mice, clean, 8-week-old and weighing 20-22 g). Human NSCLC cells A549, obtained from the American Type Culture Collection (ATCC; Manassas, VA, USA), were digested by the pancreatic enzymes and the final concentration was adjusted to $1 \times 10^{6} / \mathrm{ml}$. On day 0 , the mice $(\mathrm{n}=50)$ were narcotized with 2001 of $0.75 \%$ sodium pentobarbital solution per mouse and the subcutaneous injection were conducted of $5 \times 10^{6}$ A549 cells. The mice in the treatment group $(n=25)$ were injected with puerarin (40 $\mathrm{mg} / \mathrm{kg}$ body weight) every other day, while the mice $(n=25)$ in model group received the same volume injection of phosphate-buffered saline (PBS). After the development of a palpable tumor, the tumor volume was monitored every 6 days, briefly, tumor was isolated from five mice in each group, the volume was assessed by the following formula: tumor volume $\left(\mathrm{mm}^{3}\right)=$ maximal length $(\mathrm{mm})$ x perpendicular width $(\mathrm{mm})^{2} / 2$. All mice were assigned to euthanasia at the end of the measurements. On day 30, tumor tissues in model mice and puerarin treating mice were collected. All animal experiments were performed according to the current guidelines and under a protocol approved by the Institutional Animal Care and Use Committee.
Flow cytometry. Macrophages from local tumor tissue in model mice were considered as NSCLC model group; macrophages from lung tissues in healthy mice were considered as healthy control group; macrophages from the tumor tissue in puerarin treating mice were regarded as NSCLC model + puerarin group. The suspensions of macrophages from each group were prepared by grinding the organ through a 40-mm nylon mesh in medium. For cell surface staining, cells were stained for $20 \mathrm{~min}$ at room temperature (RT) in 1\% BSA-PBS buffer with the following panel of antibodies: Alexa Fluor 488-anti-F4/80 (Serotec, Oxford, UK) and PE-CD197, PE-iNOS, PE-CD40, PE-CD206, PE-Arg-1 and PE-CD163 (eBioscience, San Diego, CA, USA), followed by flow cytometry (BD Biosciences, San Jose, CA, USA). Data were analyzed using CellQuest software.

Western blotting. Macrophages were lysed in lysis buffer (Beyotime Institute of Biotechnology, Haimen, China) supplemented with $1 \mathrm{mM}$ phenylmethanesulfonyl fluoride (PMSF). The protein concentration was determined using the BCA protein assay (Tiangen Biotech, Co., Ltd., Beijing, China). Twenty micrograms of protein in each sample was separated by $12 \%$ SDS-PAGE and electro-transferred to polyvinylidene fluoride (PVDF) membranes (Millipore, Billerica, MA, USA) for immunoblotting. The following primary antibodies were used: anti-IFN- $\gamma$ (1:1,000, ab175878; Abcam), anti-TNF- $\alpha$ (1:1,000, ab6671; Abcam), anti-IL-12 (1:500, ab9992; Abcam), anti-IL-10 (1:500, ab34843; Abcam), anti-IL-1 $\beta$ (Santa Cruz Biotechnology, Santa Cruz, CA, USA), anti-TGF- $\beta$ (1:500, ab66043; Abcam), anti-VEGF (1:1,000, ab32152; Abcam), anti-MMP-9 (1:100, ab73734; Abcam), anti-ICAM-1 (1:20, ab20; Abcam), anti-MEK (1:20,000, ab178876; Abcam), anti-p-MEK (1:500, ab194754; Abcam), anti-ERK1/2 (1:100, ab54230; Abcam), anti-p-ERK1/2 (Santa Cruz Biotechnology) and anti-GAPDH (1:500, ab8245; Abcam), which was used as the internal reference. After incubation with the appropriate horseradish peroxidase (HRP)-conjugated secondary antibody, proteins were detected using a ChemiDoc XRS imaging system and Quantity One analysis software (Bio-Rad Laboratories, San Francisco, CA, USA).

Preparation of purified macrophages. Macrophages were prepared as previously reported (19). Macrophages from tumor tissue in model mice were seeded in 6-well tissue culture plates ( $2 \times 10^{6}$ cells/ml; Corning, Inc., Corning, NY, USA) and allowed to adhere for $1 \mathrm{~h}$. Adherent cells were purified by positive selection using CD68 MicroBeads (Miltenyi Biotec, Leiden, The Netherlands). The purity of $\mathrm{CD}^{+} 8^{+}$cells was evaluated by flow cytometry $\left(\mathrm{CD} 68^{+} \mathrm{F} 4 / 80^{+}\right.$cells $\left.>96 \%\right)$. The purified macrophages were incubated with puerarin $(40 \mu \mathrm{M})$ for $48 \mathrm{~h}$. The number of M2 phenotype $\left(\mathrm{Arg}-1^{+}\right)$was measured by flow cytometry. The level of M2 cytokines (IL-10, IL-4 and TGF- $\beta$ ) was detected by western blot analysis.

Transwell invasion assay. Transwell membranes coated with Matrigel (Becton-Dickinson, Franklin Lakes, NJ, USA) were used to assay invasion of NSCLC cells in vitro. A549 cells pretreated with or without puerarin $(40 \mu \mathrm{M})$ for $24 \mathrm{~h}$ were plated at $2 \times 10 \%$ well in the upper chamber in serum-free medium, $20 \%$ fetal bovine serum (FBS) was added to the medium in the lower chamber. After incubating for $24 \mathrm{~h}$, non-invading 

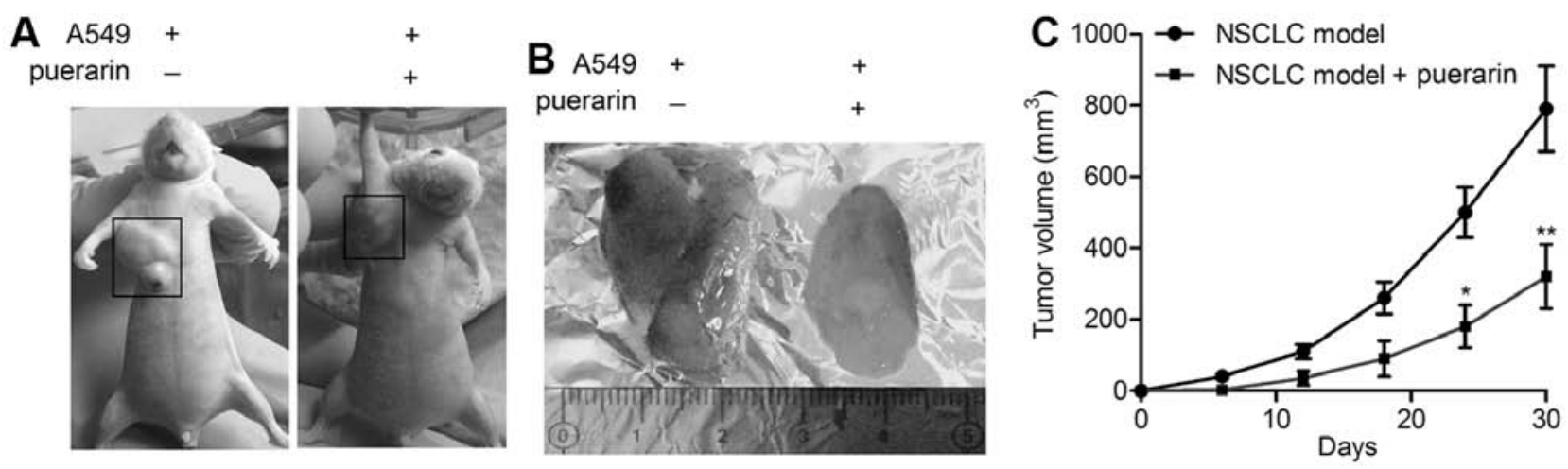

Figure 1. Puerarin reduces tumor growth in NSCLC xenograft model. NSCLC xenograft model was established by subcutaneous injection of $5 \times 10^{6}$ A549 cells into NOD/SCID mice. The mice in treatment group $(\mathrm{n}=25)$ were injected with puerarin $(40 \mathrm{mg} / \mathrm{kg}$ body weight) every other day, while the mice ( $\mathrm{n}=25)$ in model group received the same volume injection of PBS. All mice were assigned to euthanasia at the end of the measurements. (A) The tumors were isolated from 5 mice in each group every 6 days. (B and C) Volume of xenograft tumors. Data are represented as the mean \pm SD of three experiments. ${ }^{*} \mathrm{P}<0.05,{ }^{* *} \mathrm{P}<0.01 \mathrm{vs}$. NSCLC model group.

cells were removed from the top well with a cotton swab, while the bottom cells were fixed in $95 \%$ ethanol, stained with hematoxylin. The cell numbers were determined by counting the penetrating cells under a microscope at $\mathrm{x} 200$ magnification on 10 random fields in each well. Each experiment was performed in triplicate.

Wound healing assay. The CytoSelect 24-Well Wound Healing assay (Cell Biolabs, Inc., San Diego, CA, USA) was used to analyze the migration of NSCLC cells. The assay was performed according to the manufacturer's recommendations using $2 \times 10^{3}$ A549 cells pre-treated with or without puerarin $(40 \mu \mathrm{M})$ for $24 \mathrm{~h} /$ well. Image acquisition of wound fields was done after the removal of inserts $(0 \mathrm{~h})$ and wound closure documentation was completed after $24 \mathrm{~h}$ with a phase-contrast microscope (Leica DM IL; Leica Microsystems, Wetzlar, Germany) equipped with a digital camera (Leica DFC300 FX). Image analysis was conducted with Adobe Photoshop CS7 software.

Immunofluorescence staining. Purified macrophages $\left(2 \times 10^{3}\right.$ cells/well) were cultured on 8-well chamber CultureSlides (Becton-Dickinson, Bedford, MA, USA). After $8 \mathrm{~h}$, cells were fixed in $3 \%$ paraformaldehyde in PBS at room temperature for $8 \mathrm{~min}$, then permeabilized with $0.2 \%$ Triton $\mathrm{X}-100$ for $15 \mathrm{~min}$ at room temperature. After washing in PBS, the cells were incubated with primary mouse anti-ERK monoclonal antibody ( $1 \mathrm{mg} / \mathrm{ml}$; Transduction Laboratories, Lexington, KY, USA) at $4^{\circ} \mathrm{C}$ overnight. After washing, cells were incubated with biotinylated goat anti-mouse IgG (Pierce, Rockford, IL, USA) at room temperature for $1 \mathrm{~h}$. The immunoreactivity was revealed using Alexa568-conjugated streptavidin (Molecular Probes, Eugene, OR, USA) and cells were counterstained with $10 \mathrm{mg} / \mathrm{ml}$ DAPI. The cells were examined under a Nikon fluorescence microscope (Image Systems, Columbia, MD, USA).

Statistical analysis. All results are presented as mean \pm SD from a minimum of three replicates. Differences between the groups were evaluated by the SPSS version 15.0 statistical software with the Student's t-test when comparing only two groups or assessed by one-way ANOVA when more than two groups were compared. Differences were considered statistically significant at $\mathrm{P}<0.05$.

\section{Results}

Puerarin reduces tumor growth of NSCLC xenograft model. NSCLC xenograft model was established by subcutaneous injection of A549 cells into NOD/SCID mice. The representative images of NSCLC xenograft model and isolated tumors are shown in Fig. 1A and B. The tumor growth was slower in puerarin group compared with model group, and the tumor volumes were reduced significantly in NSCLC mice under treatment with puerarin $(\mathrm{P}<0.05$; Fig. 1C). The results displayed the antitumor effect of puerarin in the NSCLC xenograft model.

Puerarin inhibits macrophages polarized to M2 phenotype. TAMs are abundant components of NSCLC, the polarization status (M1/M2) has pro-inflammatory or pro-tumoral properties (20). We next explored whether the antitumor role of puerarin was involved in regulating macrophage polarization. The proportions of $\mathrm{M} 1$ markers $\left(\mathrm{CD}^{2} 7^{+}, \mathrm{iNOS}^{+}\right.$and $\left.\mathrm{CD} 40^{+}\right)$ and $\mathrm{M} 2$ markers $\left(\mathrm{CD} 206^{+}, \mathrm{Arg}-1^{+}\right.$and $\left.\mathrm{CD} 163^{+}\right)$were detectd by flow cytometry. The results showed that M1 markers were decreased in model group compared with the control group $(\mathrm{P}<0.05)$, and the treatment with puerarin in NSCLC model elevated M1 markers ( $\mathrm{P}<0.05$; Fig. $2 \mathrm{~A}$ and $\mathrm{C})$. On the contrary, M2 markers $\left(\mathrm{CD} 206^{+}\right.$, Arg- $1^{+}$and $\left.\mathrm{CD} 163^{+}\right)$were increased in model group compared with the control group $(\mathrm{P}<0.05)$, but strongly decreased adding puerarin in NSCLC model $(\mathrm{P}<0.05$; Fig. 2B and D). These results suggested that puerarin exert antitumor effect via inhibiting macrophage polarized to M2 phenotype.

Puerarin increases pro-inflammatory cytokines and decreases pro-tumor cytokines. Polarized macrophages differ in terms of effector function, cytokine and chemokine production (21). Here, the expression level of cytokines in NSCLC xenograft model was detected by western blot analysis. Levels of proinflammatory cytokines (IFN- $\gamma$, TNF- $\alpha$ and IL-12) were decreased in model group compared with the control group 


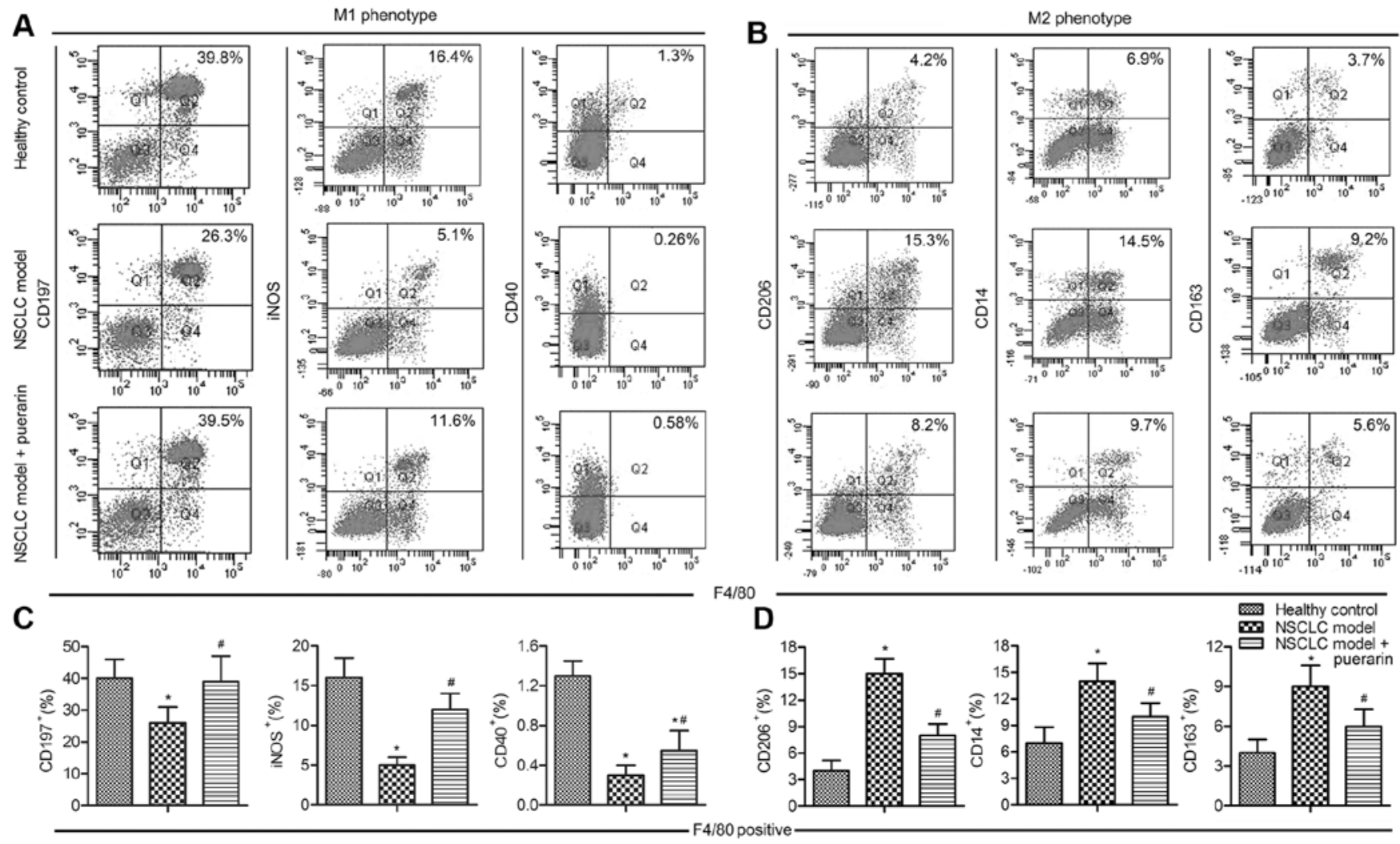

Figure 2. Puerarin inhibits macrophages polarized to M2 phenotype. On day 30, all mice were assigned to euthanasia. Tumor tissues and matched adjacent non-tumor lung tissues in model mice were collected, the tumor tissues in puerarin treating mice were also collected. Macrophages from local tumor tissue and matched adjacent non-tumor lung tissues in model mice were considered as NSCLC model group and healthy control group, respectively; macrophages from the tumor tissue in puerarin treating mice were regarded as NSCLC model + puerarin group. Cells were stained with macrophage F4/80 and M1/M2 markers, and the polarization of M1/M2 was analyzed using flow cytometry. Scatter plot, representative dot plots from one of three experiments with similar results (A and B). Histogram, quantitative results are analyzed for 5 mice in each group. Results are expressed as the percentage of double positive cells in four-quadrant diagram $(\mathrm{C}$ and $\mathrm{D})$. Data are presented as the mean $\pm \mathrm{SD}$ of three experiments. ${ }^{*} \mathrm{P}<0.05$ vs. healthy control group, ${ }^{\#} \mathrm{P}<0.05$ vs. NSCLC model group.

$(\mathrm{P}<0.05)$, but were promoted in puerarin group compared to the model group $(\mathrm{P}<0.05$; Fig. $3 \mathrm{~A})$. The expression of tumorigenesis-related cytokines (IL-10, IL-4 and TGF- $\beta$ ) was elevated in model group in comparison with the control group $(\mathrm{P}<0.05)$, but the levels were significantly reduced in NSCLC model treated with puerarin $(\mathrm{P}<0.05$; Fig. $3 \mathrm{~B})$. These results indicated puerarin exhibited antitumor effect by inflammatory cytokines and decreased tumorigenesis-related cytokines.

Puerarin acts directly on macrophages to inhibit M2 polarization. To explore whether puerarin directly acts on macrophages, we purified macrophages from model mice. An in vitro experiment was conducted to investigate the role of puerarin on M2 polarization. The results showed that the number of $\mathrm{M} 2$ phenotype (Arg- $\left.1^{+}\right)$was significantly reduced under treatment with puerarin for $24 \mathrm{~h}(\mathrm{P}<0.05$; Fig. 4A), and the number was further decreased after $48 \mathrm{~h}(\mathrm{P}<0.05$; Fig. 4B). To confirm the inhibitory effect of puerarin on M2 polarization, the level of M2 cytokines (IL-10, IL-4 and TGF- $\beta$ ) was detected by western blot analysis. The results displayed that the expression of IL-10, IL-4 and TGF- $\beta$ was strongly inhibited adding puerarin for 24 and $48 \mathrm{~h}(\mathrm{P}<0.05$; Fig. 4C-F). Taken together, our results clearly demonstrated that puerarin inhibited macrophages polarized to M2 phenotype and did not require the participation or the auxiliary of other cells.
Puerarin suppresses the invasion and migration of NSCLC cells. A report indicated that high density of M2 macrophages is associated with the metastasis in NSCLC patients (22). The effect of puerarin on the motility of NSCLC cells (A549) was measured by Transwell assays and scratch assays. The number of invasive cells was decreased by 2.5 times adding puerarin $(40 \mu \mathrm{M})$ in A549 cells $(\mathrm{P}<0.05$; Fig. $5 \mathrm{~A}$ and $\mathrm{B})$. The results of scratch assays agreed with the Transwell assays. The A549 group showed a complete closure of the gap, whereas puerarin increased the gap by $\sim 50 \%$ compared with model group $(\mathrm{P}<0.05$; Fig. $5 \mathrm{C}$ and $\mathrm{D})$. These observations suggested that puerarin was a negative metastatic regulator of NSCLC. The expression of tumor metastasis-related proteins (VEGF, MMP-9 and ICAM-1) were then determined by western blot analysis (Fig. 5E). The results indicated that the levels of the three proteins were increased in model group compared with the control group $(\mathrm{P}<0.05)$, but puerarin significantly restrained their expression compared with the model group $(\mathrm{P}<0.05$; Fig. 5F-H). The suppression of puerarin on migration-related protein expressions further confirming the inhibition effect of puerarin on the metastasis of NSCLC.

Puerarin restrains the activation of MEK/ERK1/2 pathway. To investigate the mechanism of puerarin on the metastasis of NSCLC, the level of MEK, ERK1/2 and their phosphorylated forms was measured by western blot analysis (Fig. 6A). The 
A

Pro-inflammatory cytokines
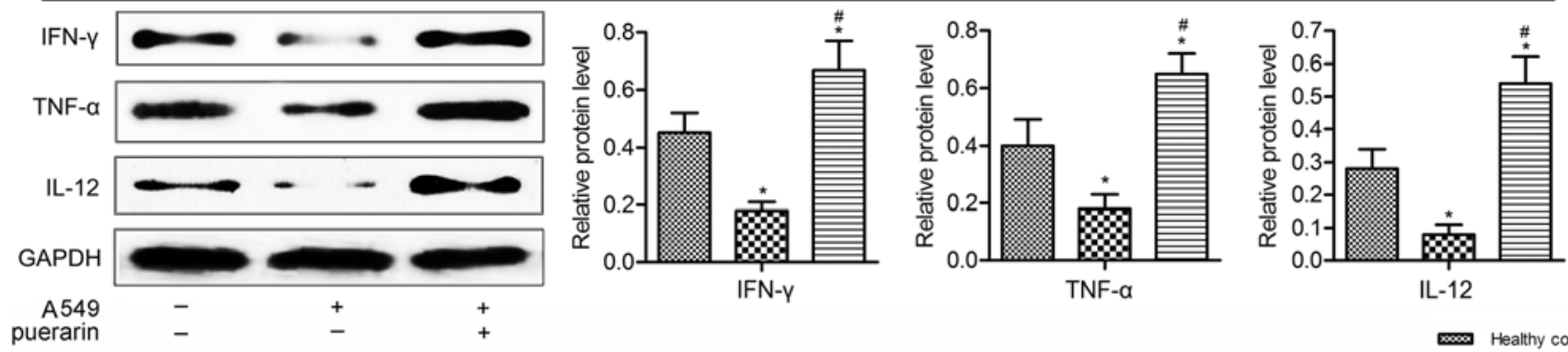

IL-12

B

Pro-tumor cytokines
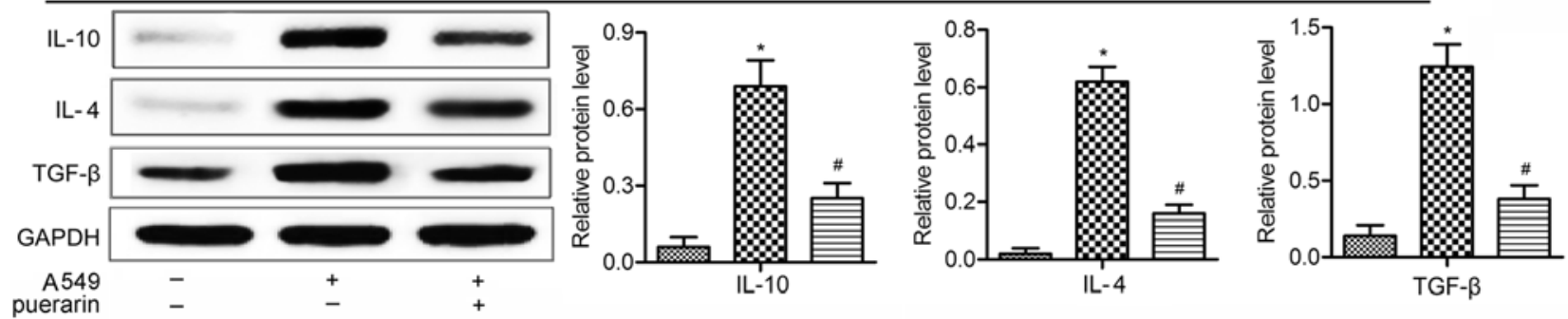

Figure 3. Puerarin increases pro-inflammatory cytokines and decreases pro-tumor cytokines. The expression of (A) pro-inflammatory cytokines (IFN- $\gamma$, TNF- $\alpha$ and IL-12) and (B) pro-tumor cytokines (IL-10, IL-4 and TGF- $\beta$ ) was detected by western blot analysis, and expressed as fold change relative to GAPDH as the loading control. Data are presented as the mean \pm SD of three experiments. ${ }^{*} \mathrm{P}<0.05$ vs. healthy control group, ${ }^{*} \mathrm{P}<0.05$ vs. NSCLC model group.
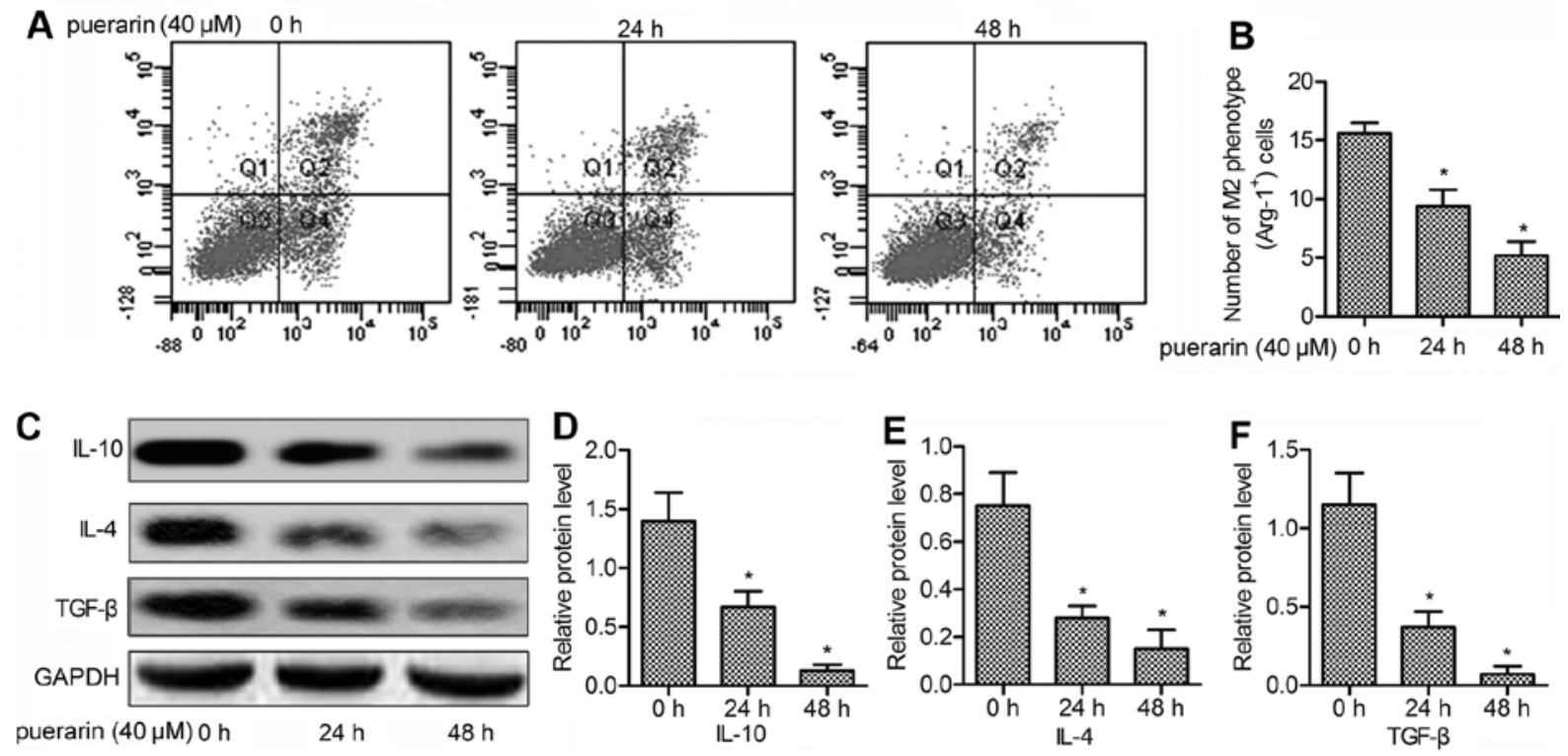

Figure 4. Puerarin acts directly on macrophages to inhibit M2 polarization. The purified macrophages were incubated with puerarin (40 $\mu \mathrm{M})$ for $48 \mathrm{~h}$. (A) The number of M2 phenotype (Arg-1 $1^{+}$) was measured by flow cytometry; (B) histogram represents the statistical analysis of Arg- $1^{+}$cells; (C) the level of M2 cytokines (IL-10, IL-4 and TGF- $\beta$ ) was detected by western blot analysis. Relative protein expressions of (D) IL-10, (E) IL-4 and (F) TGF- $\beta$ were quantified using Image-Pro Plus 6.0 software and normalized to GAPDH. The results were analyzed from three independent experiments. Data are presented as the mean $\pm \mathrm{SD}$. ${ }^{*} \mathrm{P}<0.05$ vs. macrophages treated with puerarin at $0 \mathrm{~h}$.

results displayed that the expression of p-MEK and p-ERK $1 / 2$ was enhanced in model group compared with the control group $(\mathrm{P}<0.05)$, indicating that the MEK/ERK $1 / 2$ pathway was activated in the NSCLC model. But the level of p-MEK and p-ERK $1 / 2$ was reduced significantly in NSCLC model treated with puerarin $(\mathrm{P}<0.05)$ (Fig. 6B and $\mathrm{C}$ ). To verify the inactivating role of puerarin, the subcellular localization of ERK was measured. Cells in the control group showed membrane ERK staining with minimal cytoplasmic or nuclear staining, the model group displayed predominantly nuclear ERK staining. However, the cells in the puerarin group displayed reduced cytoplasmic and nuclear staining of ERK as compared with the model group (Fig. 6D). These results indicated that puerarin restrained the activation of MEK/ERK 1/2 pathway through inhibition of the ERK nucleus translocation. 
A

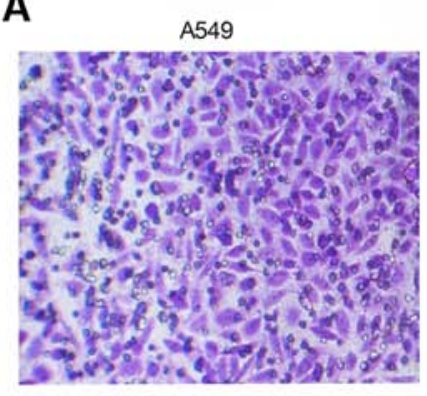

A549+ puerarin

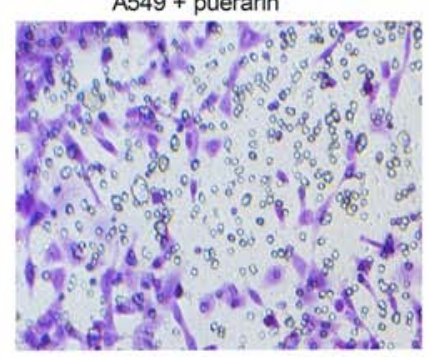

B

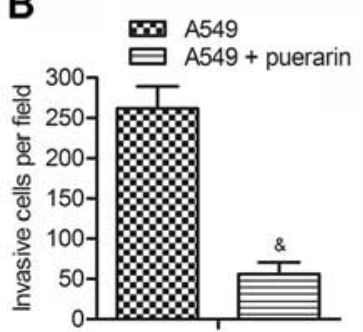

C

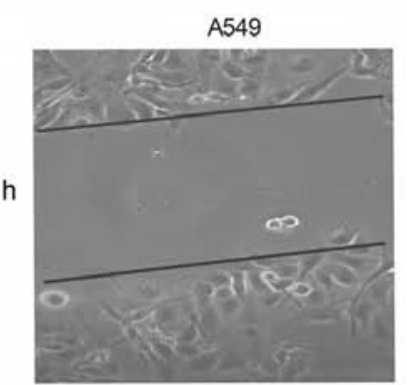

$24 \mathrm{~h}$

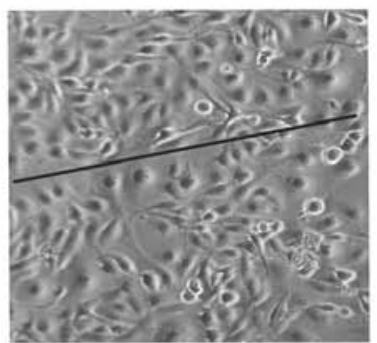

E

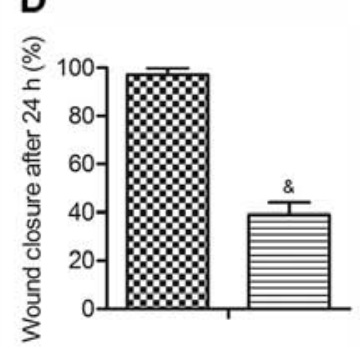

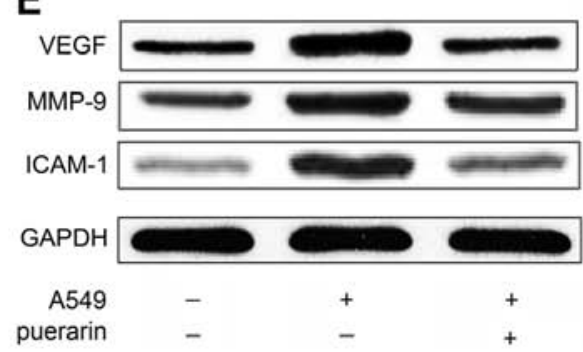

F
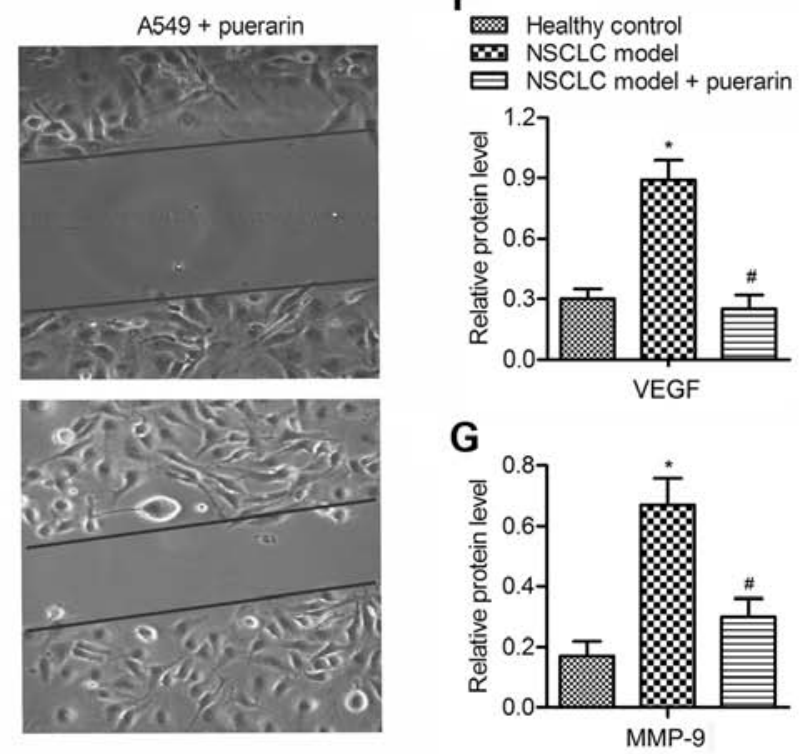

H

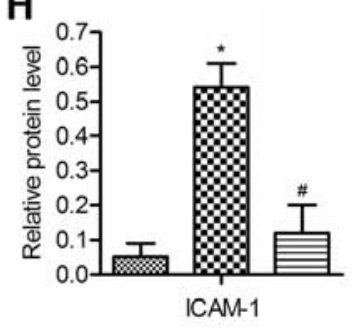

Figure 5. Puerarin suppresses the invasion and migration of NSCLC macrophages. (A) Transwell assays of A549 cells treated with puerarin for $48 \mathrm{~h}$; (B) histogram representing the Transwell assays of A549 cells in each group. (C) Wound-healing assays of A549 cells treated with puerarin for $24 \mathrm{~h}$; (D) histogram representing the wound-healing assays of A549 cells in each group. (E) The expression of tumor metastasis-related proteins in purified macrophages were detected by western blotting; (F) relative protein expression of VEGF, (G) MMP-9, (H) ICAM-1 was quantified using Image-Pro Plus 6.0 software and normalized to GAPDH. Data are presented as the mean \pm SD of three experiments. ${ }^{*} \mathrm{P}<0.05$ vs. healthy control group, ${ }^{\sharp} \mathrm{P}<0.05$ vs. NSCLC model group; ${ }^{\circledR} \mathrm{P}<0.05$ vs. A549 cells.

IL-4-induced M2 macrophage polarization and metastasis are partially offset by puerarin through MEK/ERK1/2 pathway. To convince that puerarin exerts antitumor effect on NSCLC is through inactivating MEK/ERK 1/2 pathway, we applied IL-4, which has been reported to mediate M2 macrophage polarization and promote tumor metastases via activating ERK pathway $(23,24)$. As shown in Fig. 7A and B, IL-4 upregulated the level of p-MEK and p-ERK 1/2, and a large accumulation of ERK staining in cytoplasm and nucleus was detected in NSCLC model induced by IL-4, indicating the strong activation of MEK/ERK 1/2 pathway. However, the expression of p-MEK and p-ERK 1/2 and the percentage of cells with nucleus ERK expression was significantly inhibited adding puerarin in IL-4-induced NSCLC model. These findings convinced the inhibitory effect of puerarin on MEK/ ERK 1/2 pathway. Besides, puerarin increased iNOS ${ }^{+}$macrophages (Fig. 7C), which were reduced by IL-4, whereas the elevated Arg- $1^{+}$macrophages induced by IL- 4 were downregulated by puerarin (Fig. 7D). Consistently, IL-4 reduced pro-inflammatory markers (IFN- $\gamma$, TNF- $\alpha$ and IL-12) (Fig. 7E) and increased pro-tumor markers (IL-10, IL-4 and TGF- $\beta$ ) (Fig. 7F), these effects were offset adding puerarin in IL-4 induced NSCLC model. Besides, the promoting effect of IL-4 on metastasis was restrained by puerarin (Fig. 7G). In addition, puerarin reduced the invasive numbers, slowing the wound healing (Fig. 7H) and inhibited expression of tumor metastasis-related proteins (VEGF, MMP-9 and ICAM-1) (Fig. 7I). Taken together, these results are convincing that puerarin suppressed M2 macrophage polarization and metastasis of NSCLC via inactivating MEK/ERK 1/2 pathway.

\section{Discussion}

Although early-stage non-small cell lung cancer (NSCLC) can be cured by surgical resection, a substantial fraction of patients ultimately dies due to distant metastasis (25). Therefore, identifying novel molecules that can repress the invasiveness and metastasis of NSCLC will facilitate the development of new anti-lung cancer strategies (26). As a well known isoflavone $\mathrm{C}$-glycoside, puerarin is available in common foods and has been shown to possess beneficial effects for various patients (17). The present study examined the effect of puerarin on macrophage polarization and metastasis of NSCLC.

Accumulated studies have demonstrated anticancer activity of puerarin in animal models as well as in a variety of cancer cell lines. Puerarin was suggested as a safe and effective chemosensitive agent in the treatment of human esophageal 
A
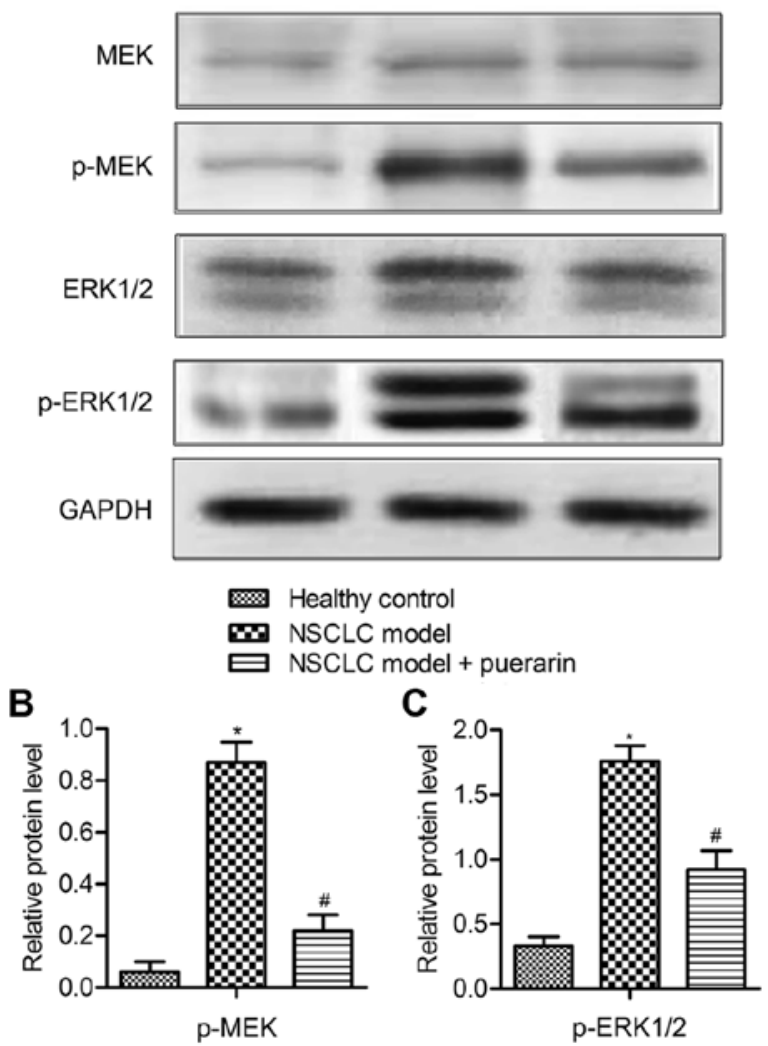

D
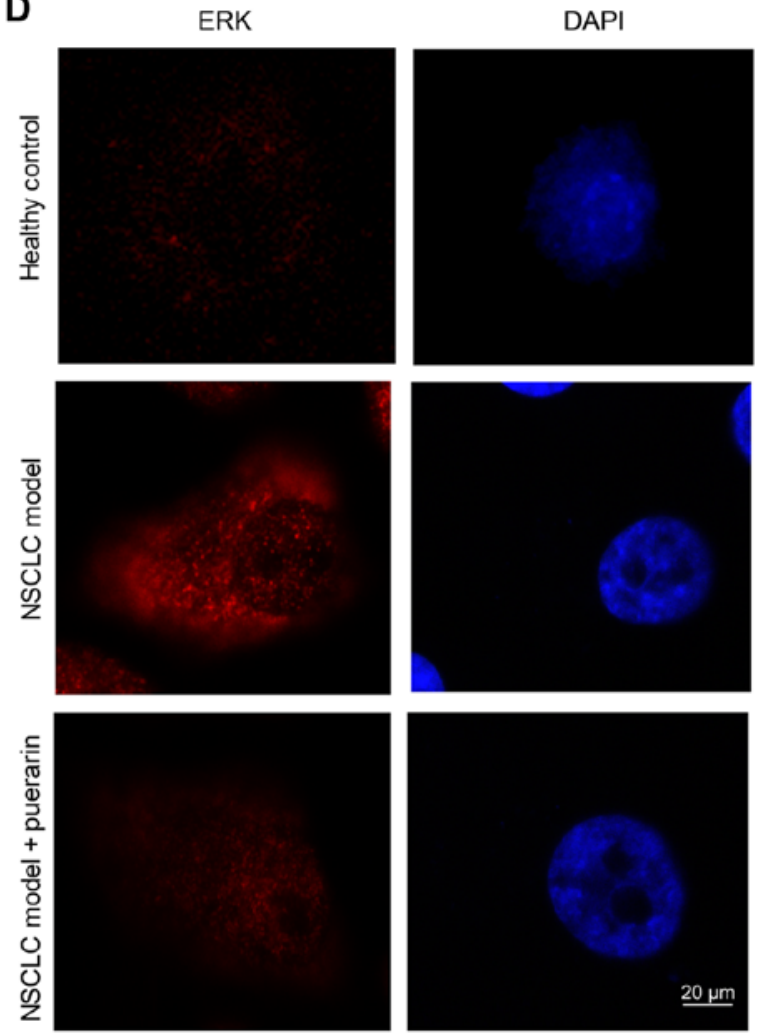

Figure 6. Puerarin restrains the activation of MEK/ERK 1/2 pathway. (A) The expression of MEK, ERK 1/2 and their phosphorylated forms was measured by western blot analysis; relative protein expression of p-MEK (B) and p-ERK (C) were quantified using Image-Pro Plus 6.0 software and normalized to GAPDH. Data are presented as the mean \pm SD of all three experiments. (D) Immunofluorescence staining of ERK in macrophages were stained for ERK and counterstained with DAPI. Data are presented as the mean \pm SD of three experiments. ${ }^{*} \mathrm{P}<0.05$ vs. healthy control group, ${ }^{\#} \mathrm{P}<0.05$ vs. NSCLC model group.

cancer, for puerarin inhibited proliferation of Eca-109 esophageal cancer cells in vitro and in vivo, and did not increase the side-effects of chemotherapy (27). The same behavior of puerarin was observed in gastric carcinoma (28), hepatocellular carcinoma (29) and colon cancer (30). Recently, an in vitro and in vivo animal study showed that puerarin significantly inhibited tumor growth in A549 cells (18). Consistently, our research exhibited that puerarin suppressed tumor growth and tumor volumes in NSCLC xenograft model, suggesting the antitumor effect of puerarin in NSCLC.

The TAMs can secrete a variety of cytokines, which play a key role in the formation of tumor microenvironment and tumor invasion and metastasis (31). The function of M1 and M2 macrophages is entirely different in the tumor microenvironment (6). To investigate the effect of puerarin on M1 and M2 marker expression in TAM populations, we selected M1 markers (e.g., CD197 and iNOS) and M2 markers (e.g., CD206 and Arg-1), based on a large amount of literature in the relevant field of macrophage polarization $(6,7,32,33)$. iNOS was suggested as an important mediator that may alter the TAM phenotype and eventually improve tumor suppressing function (34). TAMs that express iNOS were associated with extended survival in patients with NSCLC (12). A report indicated that iNOS expression was decreased in tissue from NSCLC patients with adenocarcinoma and squamous cell carcinoma compared to non-tumor tissues (7). A previous study measured reduced iNOS expression in TAMs that were directly isolated from the tumor in tumor-bearing mice (35). In accordance with these reports, our research demonstrated that M1 macrophages $\left(\mathrm{CD}_{197^{+}}\right.$, $\mathrm{iNOS}^{+}$and $\mathrm{CD}_{40}{ }^{+}$) were decreased in NSCLC model, while puerarin strongly increased M1 macrophages. These results indicated that puerarin may alter the TAM phenotype through upregulating M1 macrophages. The expression of the M2 marker in TAMs was significantly correlated to poor prognosis and lymph node metastasis in patients with advanced adenocarcinoma (14). A report exhibited that the expression of M2 marker CD163 was significantly increased in all NSCLC subtypes (7) and in patients with progressive disease (36). Similarly, the M2 markers (CD206 ${ }^{+}$, Arg- $1^{+}$and CD163 ${ }^{+}$) were increased in NSCLC model in the present study. However, puerarin strongly decreased the expression of M2 markers. Considering the percentage of TAMs within a tumor microenvironment has been linked with tumor metastasis (14). Our results suggested the anti-metastasis effect of puerarin is through elevating M1 markers and inhibiting M2 markers.

The presence of cytokines is essential for the initiation of immune responses (37). Th1 cells have been found to play a major role in anti-tumor immunity, whereas $\mathrm{Th} 2$ cells are known to act as the helper cells that influence B-cell development and produce anti-inflammatory cytokines (38). Pro-inflammatory cytokines such as TNF- $\alpha$ and IFN- $\gamma$ are known to stimulate Th1 cells $(19,39)$. In contrast, Th2 cells produce anti-inflam- 
A

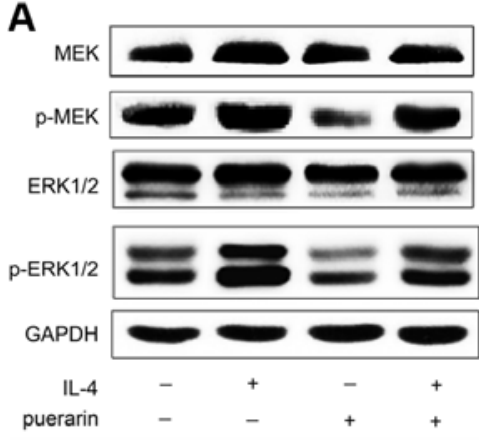

B
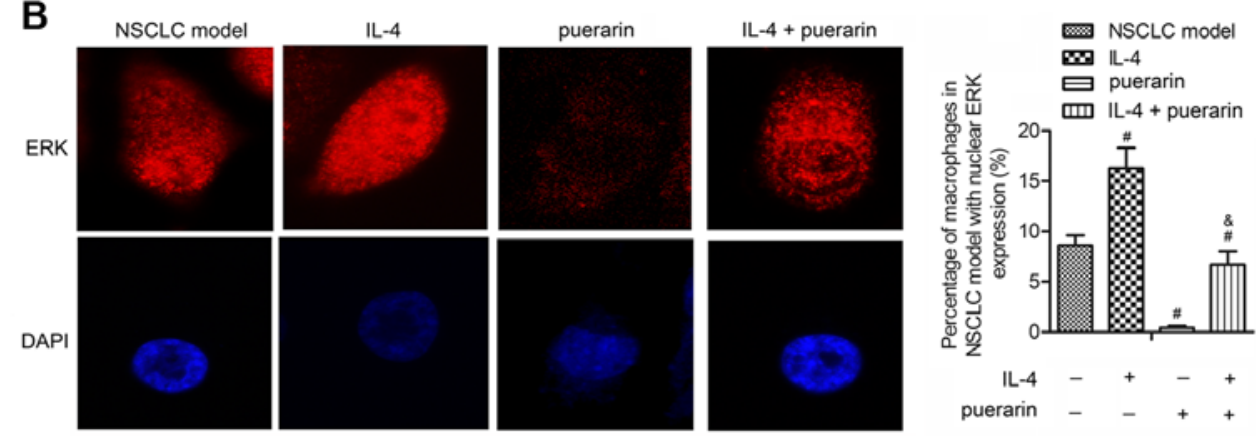

C

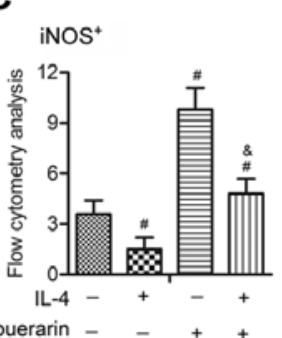

G

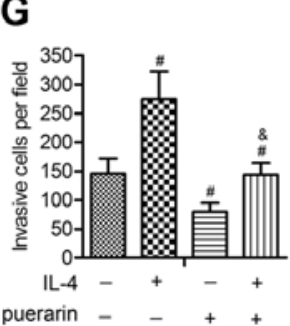

D

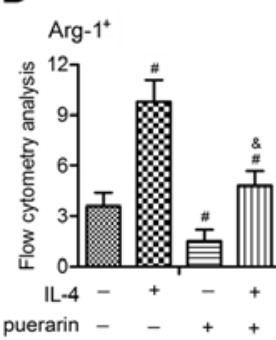

$\mathrm{H}$

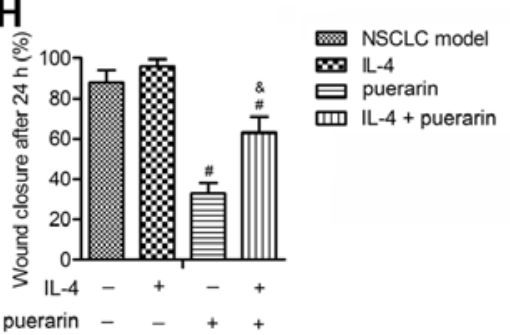

E

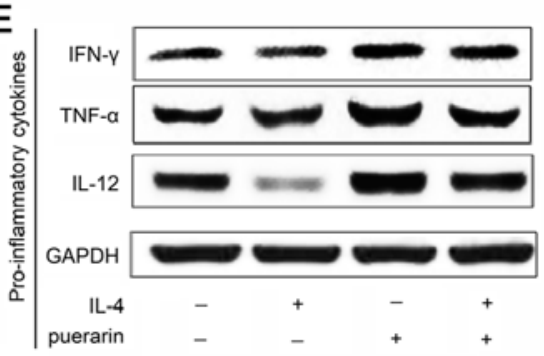

$F$

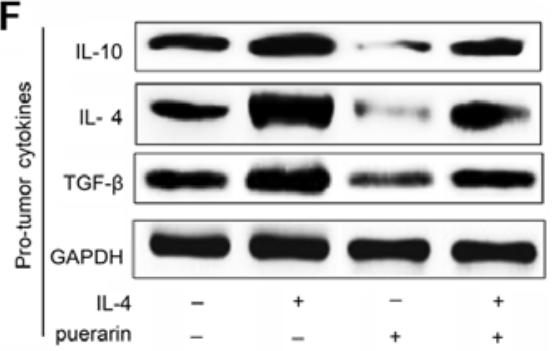

I

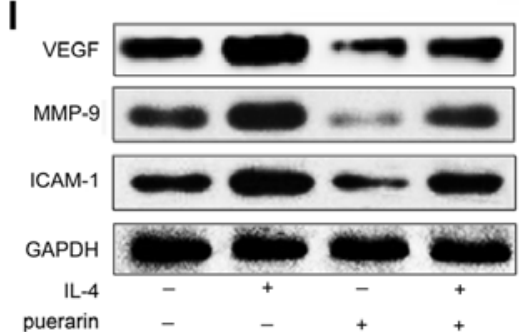

Figure 7. IL-4-induced M2 macrophage polarization and metastasis are partially offset by puerarin through MEK/ERK 1/2 pathway. Macrophages isolated from model mice and puerain treated mice were stimulated by IL-4, and were divided into four groups: NSCLC model group; IL-4 group: model mice were induced by IL-4; Puerain group: model mice were treated with puerain ( $40 \mathrm{mg} / \mathrm{kg}$ body weight); IL- 4 + puerain group: mice in puerarin group were also stimulated by IL-4. (A) The expression of MEK, ERK 1/2 and their phosphorylated forms was measured by western blot analysis; (B) immunofluorescence staining of ERK, macrophages were stained for ERK and counterstained with DAPI. Histogram presents the statistical analysis of the percentage of macrophages from NSCLC model with nuclear ERK expression; the levels of iNOS (C) and Arg-1 (D) were measured by flow cytometry; the expression of pro-inflammatory cytokines (IFN- $\gamma$, TNF- $\alpha$ and IL-12) (E) and pro-tumor cytokines (IL-10, IL-4 and TGF- $\beta$ ) (F) was detected by western blot analysis; histogram showing the Transwell assays $(\mathrm{G})$ and wound-healing assays (H) of macrophages in each group. (I) The expressions of angiogenesis factors (VEGF, MMP-9 and ICAM-1) in macrophages were detected by western blot analysis. Data are presented as the mean $\pm \mathrm{SD}$ of three experiments. ${ }^{\#} \mathrm{P}<0.05$ vs. model group, ${ }^{\&} \mathrm{P}<0.05$ vs. puerain group.

matory cytokines such as IL-4 and IL-10 (13,24). TGF- $\beta$ has been suggested to induce an M2-like phenotype characterized by upregulation of the anti-inflammatory cytokine IL-10 and downregulation of the pro-inflammatory cytokines TNF- $\alpha$ and IL-12 (40). IL-10 itself can also promote M1 to M2 transition (41). In this study, puerarin enhanced the expression of antitumor cytokines (IFN- $\gamma, \mathrm{TNF}-\alpha$ and IL-12), reduced the level of anti-inflammatory cytokines (IL-10, IL-4 and TGF- $\beta$ ). The following in vitro experiments showed that puerarin significantly reduced Arg- $1^{+}$macrophages and the level of M2 markers. These results indicated that puerarin was able to skew macrophage populations back to M1 subsets to stimulate antitumor effects within the tumor microenvironment, by directly acting on macrophages.

Puerarin can inhibit the adhesion, invasion and migration of HO-8910 cells, plays an antagonist effect against the stimulation of estrogen on the malignant behavior of tumor cells (42). A report also indicated that puerarin suppressed the tissue invasion and the vascularization of ectopic endo- metrial tissues stimulated by $17 \beta$-estradiol, by decreasing the angiogenesis factors MMP-9, ICAM-1 and VEGF (43). Based on the research, puerarin suppressed the invasion and migration of A549 cells, inhibited the expressions of tumor metastasis-related proteins (VEGF, MMP-9 and ICAM-1) in macrophages. These results confirmed that puerarin was a negative metastatic regulator of NSCLC.

The activation of MEK/ERK 1/2 pathway is closely related to NSCLC metastasis. A report indicated that the activation of ERK signaling skewing macrophage polarization away from the M1- to a tumor-promoting M2-like phenotype, and high density of M2 macrophages was associated with metastasis in NSCLC patients (22). Previous research also demonstrated that suppressing ERK1/2 led to suppression of various critical proteins for A549 invasion and migration (44). The results in this study indicated that puerarin restrained the activation of MEK/ERK 1/2 pathway through inhibition of ERK nucleus translocation. Besides, IL-4-induced M2 macrophage polarization and metastasis were partially offset by puerarin 
through restraining MEK/ERK $1 / 2$ pathway. These results are convincing that puerarin suppressed M2 macrophage polarization and metastasis of NSCLC via inactivating the MEK/ ERK 1/2 pathway.

In conclusion, the present study explored puerarin on macrophage polarization and metastasis of NSCLC. We found that puerarin suppressed tumor growth in NSCLC xenograft model, increased M1 macrophages, decreased M2 markers, enhanced the expression of antitumor cytokines, reduced the level of pro-tumor cytokines. Besides, puerarin suppressesed the invasion and migration of NSCLC macrophages, inhibited the expressions of angiogenesis factors. Finally, IL-4-induced M2 macrophage polarization and metastasis were partially offset by puerarin through restraining MEK/ERK 1/2 pathway. These results validated that puerarin is able to skew macrophage populations back to M1 subsets to stimulate anti-tumor effects, and suggest puerarin is a negative metastatic regulator of NSCLC.

\section{Acknowledgements}

All authors would like to thank the members of the Liaocheng People's Hospital, for providing helpful discussions and technical support concerning the present study.

\section{References}

1. Kim R, Emi M, Tanabe K and Arihiro K: Tumor-driven evolution of immunosuppressive networks during malignant progression. Cancer Res 66: 5527-5536, 2006.

2. Harpole DH Jr: Prognostic modeling in early stage lung cancer: An evolving process from histopathology to genomics. Thorac Surg Clin 17: 167-173, viii, 2007.

3. Wood SL, Pernemalm M, Crosbie PA and Whetton AD: The role of the tumor-microenvironment in lung cancer-metastasis and its relationship to potential therapeutic targets. Cancer Treat Rev 40: 558-566, 2014.

4. Rhee I: Diverse macrophages polarization in tumor microenvironment. Arch Pharm Res 39: 1588-1596, 2016.

5. Shigeoka M, Urakawa N, Nishio M, Takase N, Utsunomiya S, Akiyama H, Kakeji Y, Komori T, Koma Y and Yokozaki H: Cyr61 promotes CD204 expression and the migration of macrophages via MEK/ERK pathway in esophageal squamous cell carcinoma Cancer Med 4: 437-446, 2015.

6. Liu B, Wang X, Chen TZ, Li GL, Tan CC, Chen Y and Duan SQ: Polarization of M1 tumor associated macrophage promoted by the activation of TLR3 signal pathway. Asian Pac J Trop Med 9: 484-488, 2016.

7. Almatroodi SA, McDonald CF, Darby IA and Pouniotis DS: Characterization of M1/M2 tumour-associated macrophages (TAMs) and Th1/Th2 cytokine profiles in patients with NSCLC. Cancer Microenviron 9: 1-11, 2016.

8. Ferrante CJ, Pinhal-Enfield G, Elson G, Cronstein BN, Hasko G, Outram $\mathrm{S}$ and Leibovich SJ: The adenosine-dependent angiogenic switch of macrophages to an M2-like phenotype is independent of interleukin-4 receptor alpha (IL-4R $\alpha$ ) signaling. Inflammation 36: 921-931, 2013.

9. Moore SM, Holt VV, Malpass LR, Hines IN and Wheeler MD: Fatty acid-binding protein 5 limits the anti-inflammatory response in murine macrophages. Mol Immunol 67: 265-275, 2015.

10. Choi JW, Kwon MJ, Kim IH, Kim YM, Lee MK and Nam TJ: Pyropia yezoensis glycoprotein promotes the M1 to M2 macrophage phenotypic switch via the STAT3 and STAT6 transcription factors. Int J Mol Med 38: 666-674, 2016.

11. Tran TH, Rastogi R, Shelke J and Amiji MM: Modulation of macrophage functional polarity towards anti-inflammatory phenotype with plasmid DNA delivery in CD44 targeting hyaluronic acid nanoparticles. Sci Rep 5: 16632, 2015.

12. Ohri CM, Shikotra A, Green RH, Waller DA and Bradding P: Macrophages within NSCLC tumour islets are predominantly of a cytotoxic M1 phenotype associated with extended survival. Eur Respir J 33: 118-126, 2009.
13. da Silva MD, Bobinski F, Sato KL, Kolker SJ, Sluka KA and Santos AR: IL-10 cytokine released from M2 macrophages is crucial for analgesic and anti-inflammatory effects of acupuncture in a model of inflammatory muscle pain. Mol Neurobiol 51: 19-31, 2015.

14. Zhang B, Yao G, Zhang Y, Gao J, Yang B, Rao Z and Gao J: M2-polarized tumor-associated macrophages are associated with poor prognoses resulting from accelerated lymphangiogenesis in lung adenocarcinoma. Clinics (Sao Paulo) 66: 1879-1886, 2011.

15. Chen PC, Cheng HC, Wang J, Wang SW, Tai HC, Lin CW and Tang CH: Prostate cancer-derived CCN3 induces M2 macrophage infiltration and contributes to angiogenesis in prostate cancer microenvironment. Oncotarget 5: 1595-1608, 2014.

16. Zhang S, Chen S, Shen Y, Yang D, Liu X, Sun-Chi AC and $\mathrm{Xu} \mathrm{H}$ : Puerarin induces angiogenesis in myocardium of rat with myocardial infarction. Biol Pharm Bull 29: 945-950, 2006.

17. Zhou YX, Zhang $\mathrm{H}$ and Peng C: Puerarin: A review of pharmacological effects. Phytother Res 28: 961-975, 2014.

18. Chen $\mathrm{T}$, Chen $\mathrm{H}$, Wang $\mathrm{Y}$ and Zhang J: In vitro and in vivo antitumour activities of puerarin 6"-O-xyloside on human lung carcinoma A549 cell line via the induction of the mitochondriamediated apoptosis pathway. Pharm Biol 5: 1-7, 2016.

19. Liu C, Li Y, Yu J, Feng L, Hou S, Liu Y, Guo M, Xie Y, Meng J, Zhang $\mathrm{H}$, et al: Targeting the shift from M1 to M2 macrophages in experimental autoimmune encephalomyelitis mice treated with fasudil. PLoS One 8: e54841, 2013.

20. Becker M, Müller CB, De Bastiani MA and Klamt F: The prognostic impact of tumor-associated macrophages and intra-tumoral apoptosis in non-small cell lung cancer. Histol Histopathol 29: 21-31, 2014.

21. Deepak P, Kumar S and Acharya A: Interleukin-13 neutralization modulates interleukin-13 induced suppression of reactive oxygen species production in peritoneal macrophages in a murine T-cell lymphoma. Cell Immunol 251: 72-77, 2008.

22. Zhang J, Cao J, Ma S, Dong R, Meng W, Ying M, Weng Q, Chen Z, Ma J, Fang Q, et al: Tumor hypoxia enhances non-small cell lung cancer metastasis by selectively promoting macrophage M2 polarization through the activation of ERK signaling. Oncotarget 5: 9664-9677, 2014.

23. Park SJ, Lee KP, Kang S, Lee J, Sato K, Chung HY, Okajima F and Im DS: Sphingosine 1-phosphate induced anti-atherogenic and atheroprotective M2 macrophage polarization through IL-4. Cell Signal 26: 2249-2258, 2014.

24. Ferreira R, Lively S and Schlichter LC: IL-4 type 1 receptor signaling up-regulates KCNN4 expression, and increases the KCa3.1 current and its contribution to migration of alternativeactivated microglia. Front Cell Neurosci 8: 183, 2014.

25. Ji P, Diederichs S, Wang W, Böing S, Metzger R, Schneider PM, Tidow N, Brandt B, Buerger H, Bulk E, et al: MALAT-1, a novel noncoding RNA, and thymosin beta4 predict metastasis and survival in early-stage non-small cell lung cancer. Oncogene 22 : 8031-8041, 2003.

26. Shi L, Zhang B, Sun X, Lu S, Liu Z, Liu Y, Li H, Wang L, Wang X and Zhao C: MiR-204 inhibits human NSCLC metastasis through suppression of NUAK1. Br J Cancer 111: 2316-2327, 2014.

27. Wang J, Yang ZR, Guo XF, Song J, Zhang JX, Wang J and Dong WG: Synergistic effects of puerarin combined with 5-fluorouracil on esophageal cancer. Mol Med Rep 10: 2535-2541, 2014.

28. Guo XF, Yang ZR, Wang J, Lei XF, Lv XG and Dong WG: Synergistic antitumor effect of puerarin combined with 5-fluorouracil on gastric carcinoma. Mol Med Rep 11: 2562-2568, 2015.

29. Zeng YP, Yang ZR, Guo XF, Jun W and Dong WG: Synergistic effect of puerarin and 5-fluorouracil on hepatocellular carcinoma. Oncol Lett 8: 2436-2442, 2014.

30. Wang Y, Ma Y, Zheng Y, Song J, Yang X, Bi C, Zhang D and Zhang Q: In vitro and in vivo anticancer activity of a novel puerarin nanosuspension against colon cancer, with high efficacy and low toxicity. Int J Pharm 441: 728-735, 2013.

31. Ruffell B, Affara NI and Coussens LM: Differential macrophage programming in the tumor microenvironment. Trends Immunol 33: 119-126, 2012.

32. Li D, Duan M, Feng Y, Geng L, Li X and Zhang W: MiR-146a modulates macrophage polarization in systemic juvenile idiopathic arthritis by targeting INHBA. Mol Immunol 77: 205-212, 2016.

33. Yang L, Wang F, Wang L, Huang L, Wang J, Zhang B and Zhang Y: $\mathrm{CD} 63^{+}$tumor-associated macrophage is a prognostic biomarker and is associated with therapeutic effect on malignant pleural effusion of lung cancer patients. Oncotarget 6: 10592-10603, 2015. 
34. Deng X, Zhang P, Liang T, Deng S, Chen X and Zhu L: Ovarian cancer stem cells induce the M2 polarization of macrophages through the PPAR $\gamma$ and $\mathrm{NF}-\kappa \mathrm{B}$ pathways. Int J Mol Med 36: 449-454, 2015

35. Dinapoli MR, Calderon CL and Lopez DM: The altered tumoricidal capacity of macrophages isolated from tumor-bearing mice is related to reduce expression of the inducible nitric oxide synthase gene. J Exp Med 183: 1323-1329, 1996.

36. de Gaetano M, Crean D, Barry M and Belton O: M1- and M2-type macrophage responses are predictive of adverse outcomes in human atherosclerosis. Front Immunol 7: 275, 2016.

37. Belardelli F and Ferrantini M: Cytokines as a link between innate and adaptive antitumor immunity. Trends Immunol 23: 201-208, 2002.

38. Seo N and Tokura Y: Downregulation of innate and acquired antitumor immunity by bystander gammadelta and alphabeta $\mathrm{T}$ lymphocytes with Th2 or Tr1 cytokine profiles. J Interferon Cytokine Res 19: 555-561, 1999.

39. Iwamoto S, Iwai S, Tsujiyama K, Kurahashi C, Takeshita K, Naoe M, Masunaga A, Ogawa Y, Oguchi K and Miyazaki A: TNF-alpha drives human $\mathrm{CD} 14^{+}$monocytes to differentiate into $\mathrm{CD} 70^{+}$dendritic cells evoking Th1 and Th17 responses. J Immunol 179: 1449-1457, 2007.
40. Zhang F, Wang H, Wang X, Jiang G, Liu H, Zhang G, Wang H, Fang R, Bu X, Cai S, et al: TGF- $\beta$ induces M2-like macrophage polarization via SNAIL-mediated suppression of a pro-inflammatory phenotype. Oncotarget 13: 10561, 2016.

41. Deng B, Wehling-Henricks M, Villalta SA, Wang Y and Tidball JG: IL-10 triggers changes in macrophage phenotype that promote muscle growth and regeneration. J Immunol 189: 3669-3680, 2012.

42. Han J, Yu CQ and Shen W: Inhibitory effects of puerarin on invasion and metastasis of oophoroma cells HO-8910. Zhongguo Zhong Xi Yi Jie He Za Zhi 29: 632-635, 2009 (In Chinese).

43. Wang D, Liu Y, Han J, Zai D, Ji M, Cheng W, Xu L, Yang L, He M, Ni J, et al: Puerarin suppresses invasion and vascularization of endometriosis tissue stimulated by $17 \beta$-estradiol. PLoS One 6: e25011, 2011.

44. Lee SH, Jaganath IB, Manikam R and Sekaran SD: Inhibition of Raf-MEK-ERK and hypoxia pathways by Phyllanthus prevents metastasis in human lung (A549) cancer cell line. BMC Complement Altern Med 13: 271, 2013. 